\title{
Exploitation des végétaux et artisanat textile au Néolithique final sur les sites de la vallée de la Deûle (Nord-Pas-de-Calais)
}

Emmanuelle Martia/*

1.
Palynologie : Muriel Boulen (InRAP et UmR 7041);
carpologie : Marie-France Dietsch-Sellami
(INRAP et UMR 6566); tracéologie du silex
et expérimentation : Jean-Paul Caspar †;
anthracologie : Sylvie Coubray (INRAP
et UMR 7041); pédologie : Kai Fechner (INRAP);
expérimentation : Philippe Féray (INRAP);
étude de l'industrie en os : Yolaine Maigrot
(UmR 7041); étude de l'industrie lithique
et responsable de fouilles : Emmanuelle Martial;
étude de la céramique et responsable de fouilles:
Ivan Praud (INRAP et UMR 7041).

2.

À partir de 2008, ce programme constitue un axe de recherche collective de L'INRAP intitulé "Le Néolithique final dans le nord de la France: une approche pluridisciplinaire des sites

du II $^{\mathrm{e}}$ millénaire avant notre ère, entre la Deûle et l'Escaut" (resp. E. Martial et I. Praud).
$\mathrm{L}$ 'exploitation de matières végétales pour la production textile est relativement bien documentée sur les stations lacustres néolithiques du Jura et de Suisse (Médard 2000 et 2006). Dans le nord de la France, aucun vestige textile proprement dit n'a encore été découvert en contexte préhistorique : aucune pelote de fil, aucun fragment d'étoffe. Les données acquises sur des occupations néolithiques fouillées ces dernières années dans les départements du Nord et du Pas-de-Calais permettent une approche pluridisciplinaire relativement complète, grâce au bon état de conservation des vestiges trouvés, en contexte humide parfois. L'apport des analyses archéologiques, pédologiques, paléobotaniques et fonctionnelles, complétées par les sources ethnohistoriques et l'expérimentation, permet d'aborder la question de l'exploitation des végétaux à des fins textiles. L'approche croisée s'effectue grâce à une collaboration étroite entre les archéologues et les différents spécialistes ${ }^{1}$ à toutes les étapes de la démarche, depuis le terrain jusqu'à l'analyse des contextes et les études de mobilier. Cette présentation est donc le fruit d'un travail collectif dont les protagonistes sont désormais réunis au sein d'un programme de recherche collective ${ }^{2}$.

La présence récurrente d'outils employés pour la production textile (fusaïoles et poids de métier à tisser) sur ces occupations de la fin du Néolithique fait écho à certaines espèces végétales identifiées parmi les pollens et les paléosemences conservés dans le comblement de structures archéologiques parfois singulières. Différents aspects du travail des fibres végétales sont attestés, qui illustrent autant d'étapes distinctes de la chaîne opératoire textile. Une réflexion à propos de leur distribution spatiale sur l'aire géographique considérée sera enfin proposée.

Un secteur d'étude privilégié pour la fin du Néolithique : la vallée de la Deûle

Les découvertes réalisées ces huit dernières années dans le cadre des opérations d'archéologie préventive ont considérablement enrichi la documentation sur le III ${ }^{\mathrm{e}}$ millénaire avant J.-C. dans le Nord-Pas-de-Calais. Notre propos concerne ici plus particulièrement les principales occupations qui bordent la Deûle et apportent des éléments inédits au «Deûle-Escaut", groupe régional du Néolithique final (fig. 1).

Cette rivière s'écoule du sud vers le nord jusqu'à sa confluence avec la Lys, à la frontière belge. Son lit, aujourd'hui canalisé, décrivait jadis de larges méandres dans une vallée marécageuse au relief très peu marqué, envahie de dépôts éoliens quaternaires d'épaisseur moindre sur la rive droite.

Situés à 10-15 km au sud de Lille, les gisements sont localisés sur la bordure ouest du dôme de craie blanche à silex (Sénonien) du Mélantois, tous sur la rive droite au contact avec le fond de vallée marécageux. Deux d'entre eux, distants de $700 \mathrm{~m}$, sont situés à Houplin-Ancoisne, "rue Marx Dormoy" et au "Marais de Santes», un autre se trouve à Annoeullin "rue Lavoisier" et le quatrième à Carvin "zone industrielle du Château" (Martial, Praud et Bostyn 2004). Ces quatre occupations sont sub-contemporaines, datées par le radiocarbone et/ou la dendrologie de la première moitié du mile millénaire cal BC.

L'environnement dans lequel ces populations ont choisi de s'implanter à la fin du Néolithique paraît favorable à divers titres. Au contact entre deux biotopes distincts - le versant crayeux recouvert de limons d'une part, le fond de la vallée marécageux d'autre part -, il se caractérise par une grande diversité des ressources immédiatement 


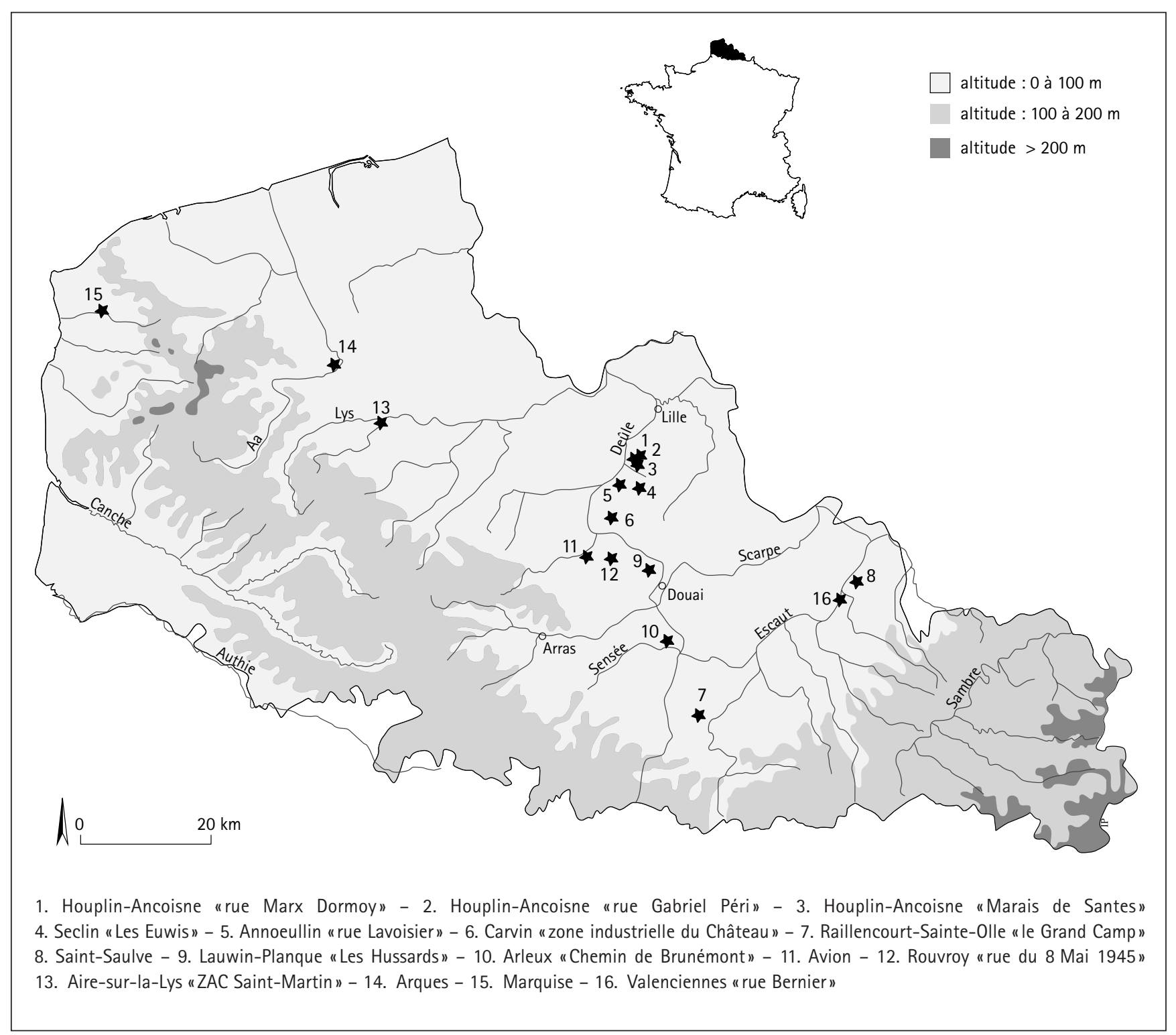

Fig. 1 - Localisation des sites du Néolithique final dans le Nord - Pas-de-Calais.

accessibles : celles propres au marais et à la rivière (eau, faune et végétation spécifiques) et celles qui caractérisent le versant boisé couvert de limons fertiles favorables, en particulier, à la culture des céréales et offrant un accès aux gîtes de matières siliceuses de bonne qualité.

Les deux sites d'Houplin-Ancoisne se singularisent par des aménagements structurés hors du commun. L'occupation du "Marais de Santes" (fig.2) comprend plusieurs constructions sur poteaux de bois dont l'élément principal est un bâtiment monumental de 43,50 m de long sur 12,80 m de large, élevé à l'intérieur d'une palissade curviligne (Praud et al. 2007). La portion de site fouillée «rue Marx Dormoy" à Houplin-Ancoisne comprend notamment une très grande fosse creusée à l'intérieur d'une puissante palissade (Martial et Praud sous presse). Les deux autres occupations sont plus «modestes». Celle de la "zone industrielle du Château" à Carvin, située au sommet d'une petite butte limoneuse dominant une vaste zone marécageuse, comprend 6 fosses détritiques à caractère domestique réparties à proximité d'un bâtiment sur poteaux partiellement dégagé sur l'emprise (Martial, Praud et Bostyn 2004). Enfin, le site d'Annoeullin "rue Lavoisier» se caractérise par la conservation du niveau archéologique dans les anciennes dépressions colmatées; de fortes concentrations de mobilier néolithique correspondaient à des aires d'activité et de rejets indiquant vraisemblablement la proximité immédiate d'un habitat structuré en dehors de l'emprise accessible (Praud et Martial 2000).

\section{Les vestiges paléobotaniques}

Les analyses palynologiques, carpologiques et anthracologiques livrent des informations sur les espèces végétales, sauvages et cultivées, exploitées par les premières sociétés 


\section{E. Martial | Exploitation des végétaux et artisanat textile au Néolithique final}

agro-pastorales. Certaines se caractérisent par la présence de fibres de tige ou d'écorce qui ont pu être employées pour l'artisanat textile. Les fibres recueillies sur la surface interne de l'écorce de certains arbres constituent le liber.

L'étude dendrologique des restes architecturaux (poteaux, cuvelage de puits) conservés au "Marais de Santes» à HouplinAncoisne montre que tous les bois d'œuvre étaient en chêne (Quercus sp.), certains atteignant des diamètres exceptionnels (Praud et al. 2007). Si nous postulons que les troncs étaient écorcés avant d'être utilisés entiers ou débités, il est alors possible d'évaluer la quantité de liber disponible, proportionnelle à un effectif de plusieurs dizaines voire centaine(s) d'arbres abattus pour la construction de la palissade et des bâtiments colossaux.

De même, le tilleul (Tilia sp.) est fortement représenté dans les spectres polliniques du site de la "rue Marx Dormoy" à Houplin-Ancoisne.

Sur ces mêmes sites, l'abondance des plantes sauvages aquatiques ou de bord de rivière susceptibles d'être exploitées pour fournir des fibres textiles est également enregistrée. C'est le cas du roseau (Phragmites australis), des graminées et de l'ortie (Urtica dioica L.).

Les analyses anthracologiques effectuées sur les occupations néolithiques de la vallée de la Deûle révèlent également l'utilisation de différentes essences d'arbres parmi lesquelles le bouleau (Betula sp.) et le saule (Salix sp.) - outre le chêne et le tilleul déjà cités - qui recèlent du liber dont l'usage textile au Néolithique est fréquemment identifié (Médard 2000).

Enfin, le lin cultivé (Linum usitatissimum L.), espèce emblématique de la production textile dont les plus anciens vestiges connus datent du $v^{\mathrm{e}}$ millénaire (Médard 2006), a été identifié à Houplin-Ancoisne : quelques rares pollens ont été déterminés sur les deux sites mais le nombre de graines carbonisées et surtout leur fréquence sont particulièrement élevés "rue Marx Dormoy" (tabl. 1).

Tabl. 1 - Carpologie du site d'Houplin-Ancoisne "rue Marx Dormoy " (D'APRÉS M.-F. DieTSCH-SelLAMI)

\begin{tabular}{|l|c|}
\hline Plantes cultivées & Fréquences \\
\hline Hordeum vulgare & $25 \%$ \\
Hordeum vulgare var. nudum & $12,5 \%$ \\
Panicum miliaceum & $12,5 \%$ \\
Pisum sativum & $12,5 \%$ \\
Linum usitatissimum & $25 \%$ \\
Triticum aestivo-compactum & $25 \%$ \\
Triticum dicoccum & $50 \%$ \\
Triticum monococcum & $37,5 \%$ \\
\hline
\end{tabular}

La palette d'espèces végétales, sauvages ou cultivées, capables de fournir la matière première nécessaire à la production textile est riche et variée, mais l'absence de restes textiles conservés et l'état actuel de la recherche ne permettent pas de trancher en faveur de l'utilisation d'une espèce plutôt qu'une autre. Cependant, à partir des données archéobotaniques, on peut envisager une situation comparable à celle mise en évidence sur les sites lacustres du Jura ou de Suisse, où la conservation des matières organiques montre la fabrication conjointe de fils à partir de fibres de liber et de fibres de lin, ces dernières s'avérant minoritaires parmi les échantillons analysés (Médard 2000).

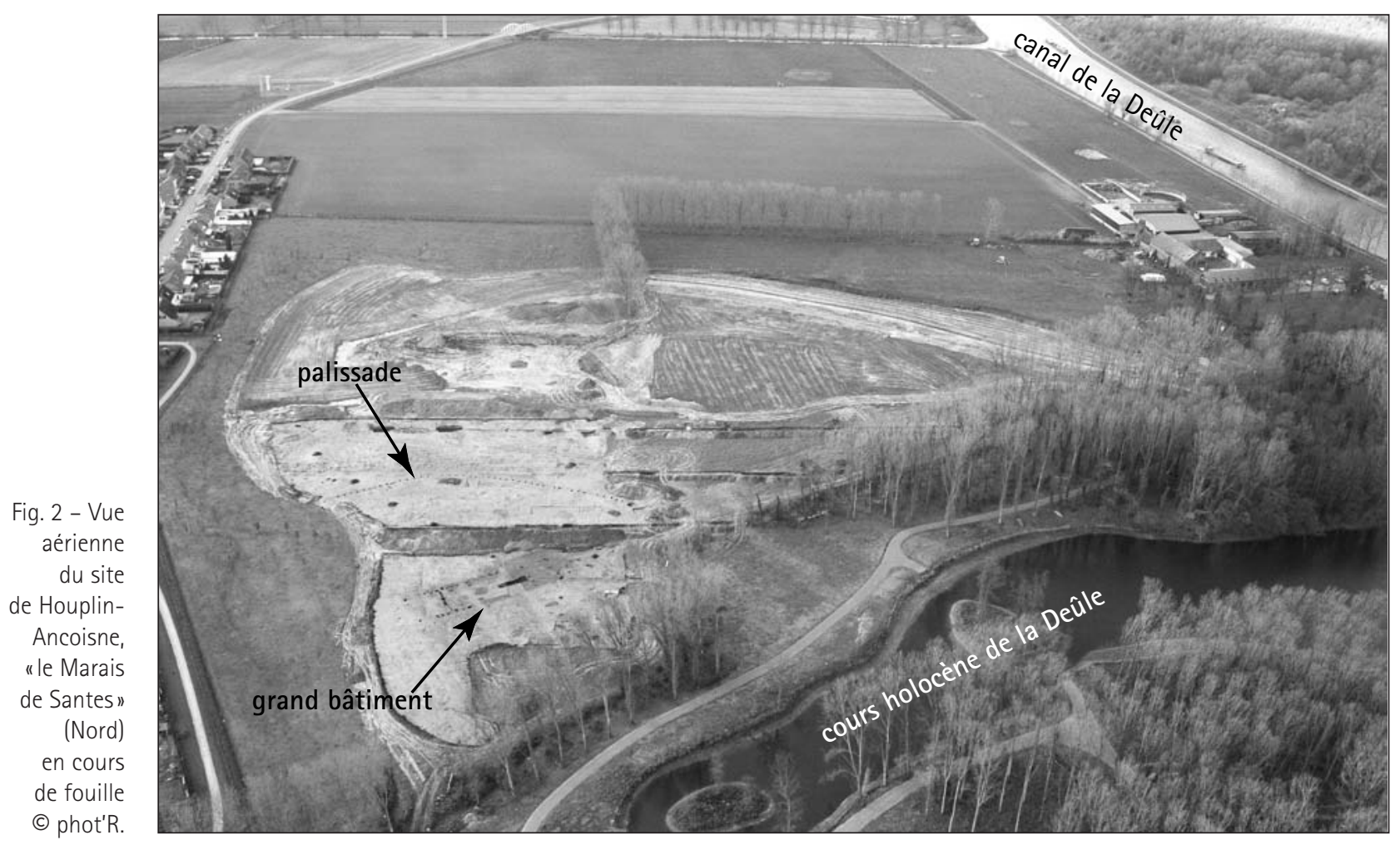




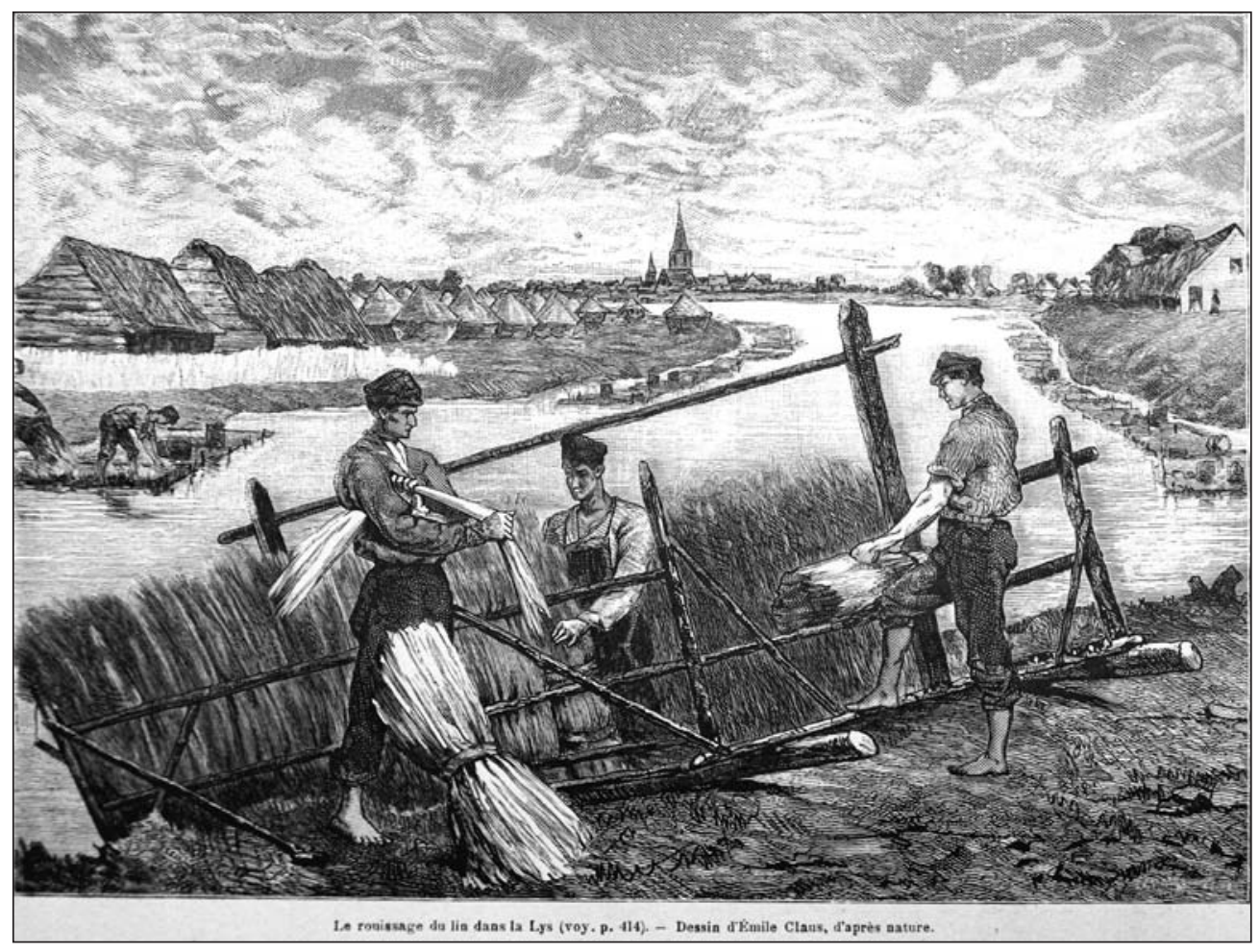

Fig. 3 - Le rouissage dans les eaux de la Lys (gravure ancienne).

fibres de lin ou de dissocier les couches de liber. Ce processus bactériologique (fermentation) doit être contrôlé de manière à ce que les fibres restent intactes et solides.

Le rouissage s'effectue en eau courante ou stagnante en immergeant les tiges ou le liber, ou bien à l'air libre sous l'action combinée du soleil et de la pluie.

Traditionnellement, en Belgique et dans le nord de la France, pour le rouissage du lin, on faisait tremper les

Les indices de préparation des fibres textiles

Grâce à la documentation ethno-historique (Musée national du lin à Courtrai, Belgique), les procédés artisanaux d'obtention et de transformation des fibres végétales utilisées pour la production textile sont bien connus et illustrés. Les méthodes d'exploitation d'une matière première comme le lin par exemple, dont la qualité est particulièrement renommée dans nos régions d'Europe occidentale, ont très peu évolué jusqu'au XIX ${ }^{\mathrm{e}}$ siècle où la production textile est entrée dans l'industrialisation. Qu'il s'agisse du lin ou de toute autre plante dont la tige comporte des fibres, les schémas opératoires d'acquisition de la matière première sont semblables et comprennent une succession d'étapes - récolte, égrenage, rouissage, séchage, battage, teillage et peignage - préalables à sa transformation proprement dite - filage et tissage (Martial et Médard 2007).

Les modalités d'acquisition et de préparation de la matière première sont difficiles à mettre en évidence car elles laissent très peu de traces archéologiques; elles ne nécessitent, en effet, pas ou peu d'outils. Toutefois, deux catégories de données distinctes permettent de penser que le rouissage et le teillage ont pu être pratiqués à Houplin-Ancoisne : l'interprétation d'une structure archéologique singulière d'une part, et les résultats de l'analyse fonctionnelle de l'outillage en silex d'autre part.

Une fosse de rouissage à Houplin-Ancoisne, "rue Marx Dormoy"?

Le rouissage est l'opération la plus importante et la plus délicate dans la préparation des fibres textiles; la qualité de la filasse et son aptitude au filage dépendent de ce traitement qui consiste à faire macérer les plantes afin d'éliminer la pectose soudant les bottes dans la rivière (fig. 3). La décomposition bactérienne donnait à l'eau une couleur rousse et dégageait une odeur nauséabonde : "Les eaux de la Loire, en France, mais surtout celles de la Lys, en Belgique, sur une longueur de $75 \mathrm{~km}$, de Warneton à Deynze, ont des qualités spéciales qui donnent aux filasses une finesse et une ténacité extraordinaire." (Constantin et Faideau 1922) Jusqu'au $\mathrm{Xx}^{\mathrm{e}}$ siècle, dans certaines régions comme la Picardie ou la Lorraine, on faisait rouir le lin ou le chanvre dans des fosses creusées en zone humide et drainées par un ruisseau, appelées rouissoirs, routoirs ou encore roises (fig. 4). Ces techniques sont de nos jours interdites pour des raisons environnementales. Le rouissage à l'eau donnait cependant une toile plus blanche et un résultat moins aléatoire que le rouissage à l'air tributaire de la météo.

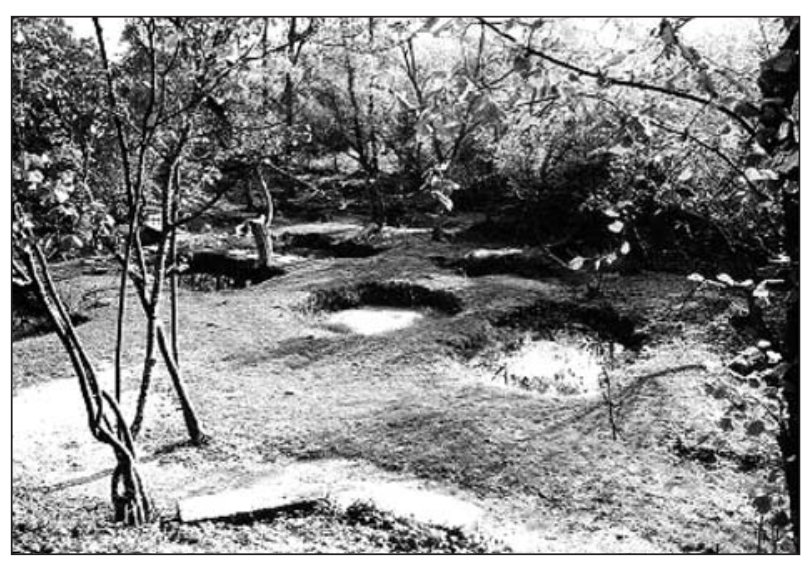

Fig. 4 - Vue actuelle de fosses destinées au rouissage du chanvre à Lucey (Lorraine), encore en fonction au Xxe siècle @ Les Mermet. 


\section{E. Martial | Exploitation des végétaux et artisanat textile au Néolithique final}

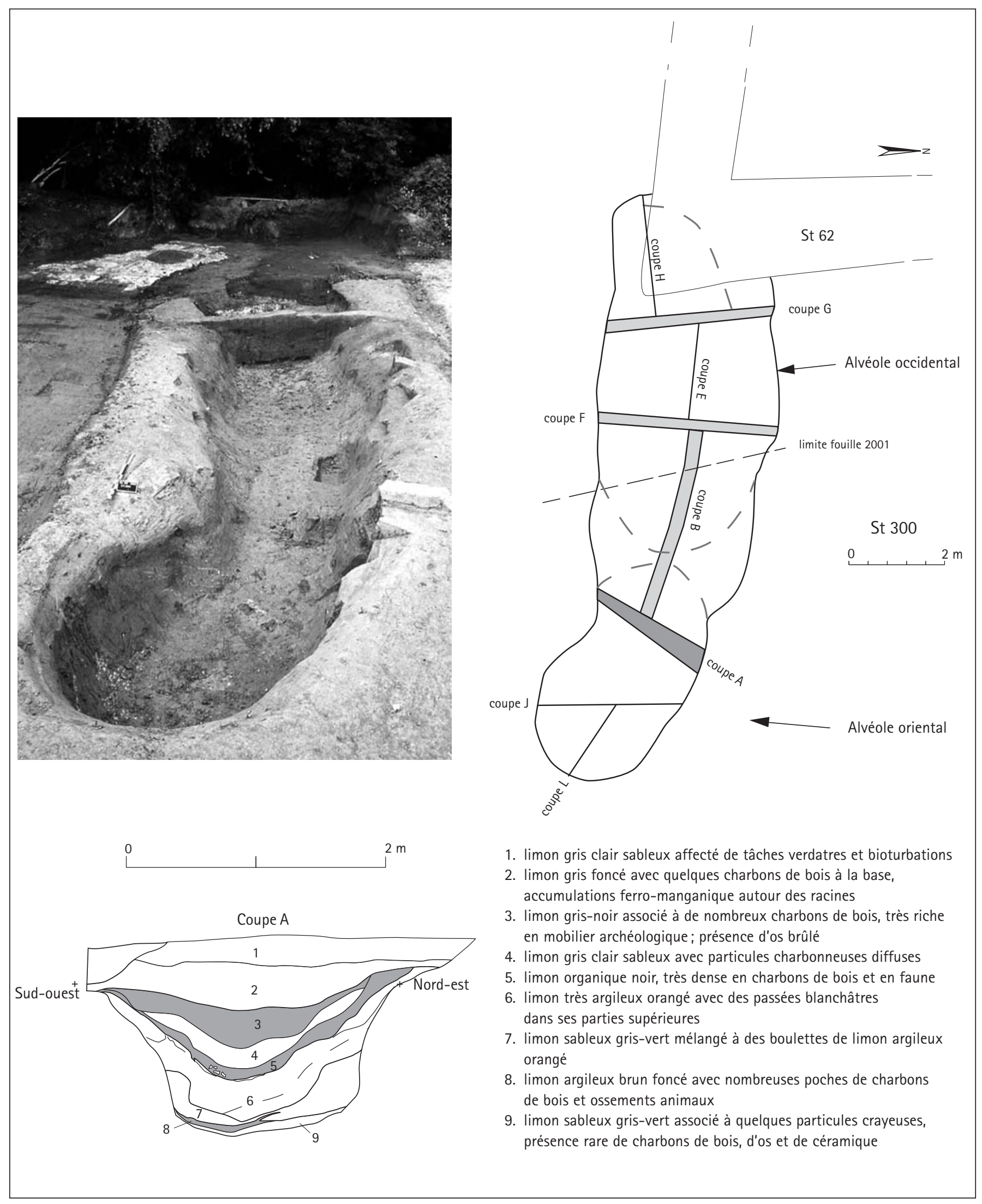

Fig. 5 - La grande fosse du site de Houplin-Ancoisne "rue Marx Dormoy» (Nord) : plan, relevé d'une coupe stratigraphique et vue de la fosse en fin de fouille (cliché I. Praud). 

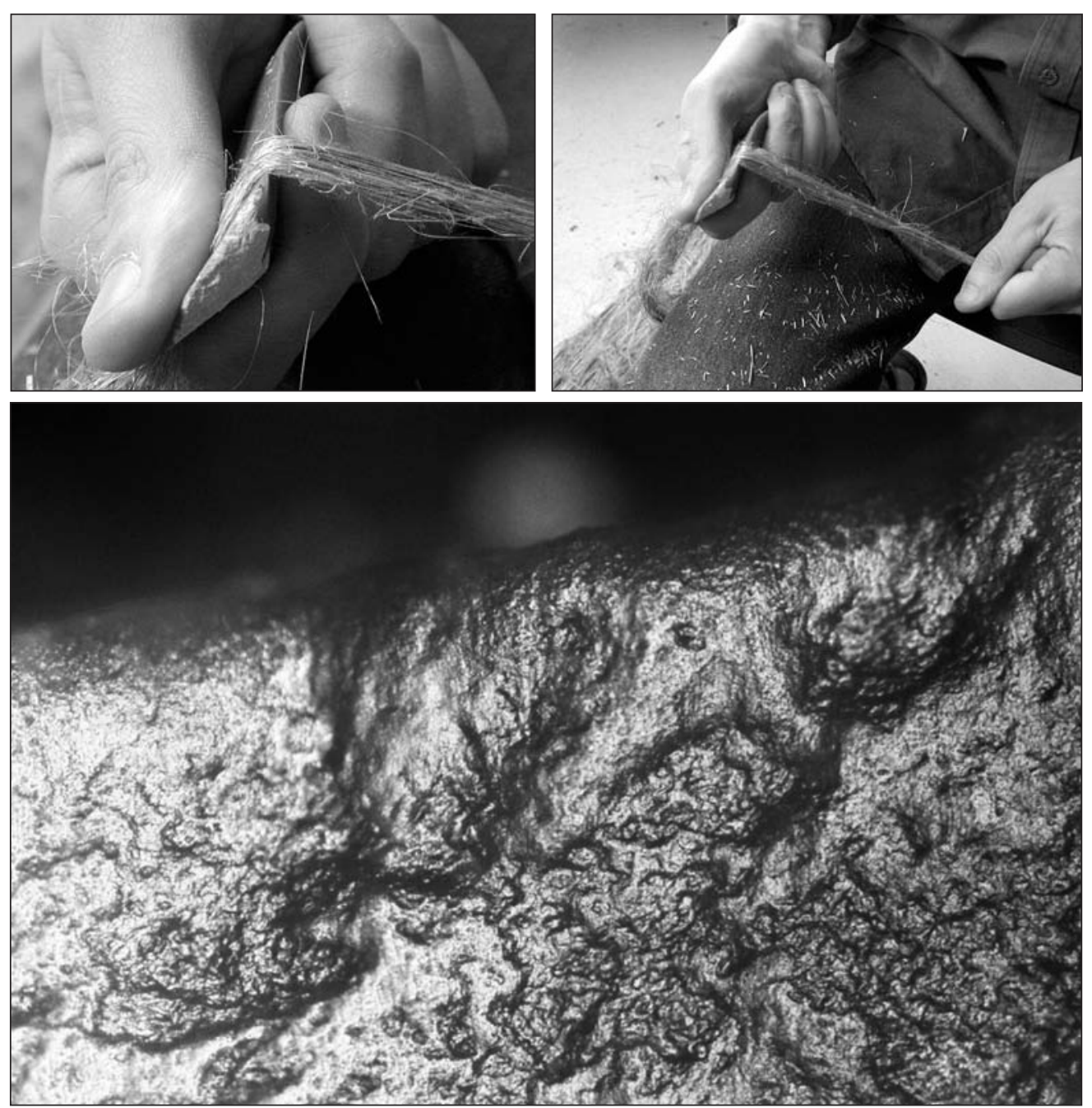

À Houplin-Ancoisne, "rue Marx Dormoy ", une très grande fosse creusée dans la zone basse du site soumise aux battements de la nappe phréatique constitue un "ensemble clos" dont les couches successives sont parfaitement datées par le radiocarbone. Mesurant 12,40 m de long sur 3,20 m de large et $1,60 \mathrm{~m}$ de profondeur maximale (soit un volume d'environ $55 \mathrm{~m}^{3}$ ), cette fosse a un profil en "U» caractérisé par un fond plat et des parois sub-verticales. Elle comprenait deux alvéoles pour lesquels au moins douze épisodes ont été identifiés dans le comblement stratifié et complexe (fig. 5). Ces sédiments ont livré, entre autres restes, un pollen et des graines carbonisées de lin cultivé (Linum usitatissimum) associés à des pollens et à du liber de tilleul carbonisé (Tilia sp.), ainsi qu'à quelques pollens de roseau (Typha angustifolia) et de cypéracées (joncs). Le mobilier comprend notamment des fusaïoles et des fragments de pesons en terre cuite. Les analyses chimiques, pédologiques et micromorphologiques révèlent l'existence de fins dépôts de restes organiques dans le fond et sur les parois de la fosse (végétaux en attente d'identification), d'eau stagnante, ainsi qu'un processus d'acidification du sédiment accompagné d'un enrichissement exceptionnel en phosphates - caractères tous compatibles avec une activité de rouissage. Des
Fig. 6 - Teillage expérimental de tiges de lin rouies et sèches à l'aide d'un bord brut d'éclat en silex et détail de l'usure expérimentale observée au microscope (grossissement $200 \mathrm{x}$ ) (clichés E. Martial et J.-P. Caspar).

phénomènes comparables ont été observés dans une fosse géante médiévale à Esplechin "Maraîche" (Hainaut, Belgique), interprétée comme possible fosse de rouissage (Fechner 2000 : 95-97). Dans la fosse d'Houplin-Ancoisne, la présence de dépôts d'ossements d'animaux, restes de consommation et de boucherie, qui tapissaient littéralement le fond et les parois reste encore difficilement interprétable.

\section{L'analyse fonctionnelle de l'outillage en silex}

L'expérimentation etl'analyse fonctionnelle d'outils archéologiques livrent des résultats particulièrement intéressants sur la possibilité de teiller des fibres de lin à l'aide de bords bruts d'éclats ou de lames de silex. Le but d'une telle opération est de débarrasser la filasse des fragments résiduels de bois de la tige (les anas) qui restent accrochés. L'hypothèse et le protocole expérimental sont inspirés en particulier par la morphologie des racloirs employés pour ce traitement (Musée national du lin de Courtrai). L'extrémité d'un faisceau d'une dizaine de tiges de lin sèches préalablement rouies est tenue dans une main. L'autre main fait glisser l'outil en silex transversalement en exerçant une légère pression du pouce, de manière à ce que le bord racle la filasse sur toute sa longueur. Ce procédé, très efficace et peu traumatisant pour la fibre textile, permet aux anas de se détacher facilement et entraîne la formation rapide d'étoupe. L'observation des outils expérimentaux sous le microscope a montré la formation rapide d'un micropoli marginal mat, souligné de stries dues à la présence de particules abrasives (terre, poussière) sur les tiges (fig.6). Ce poli, par son modelé et sa texture, ressemble fortement aux traces produites par le raclage de peaux sèches à l'état souple. Il a cependant été clairement identifié sur certains bords d'outils en silex du site d'Houplin-Ancoisne, "rue MarxDormoy». Les résultats de cette expérimentation incitent le tracéologue à 


\section{E. Martial | Exploitation des végétaux et artisanat textile au Néolithique final}

réviser l'interprétation de certains polis d'usure attribués au travail de la peau et à réévaluer l'importance de l'exploitation des végétaux dans le contexte néolithique européen (Caspar, Féray et Martial 2005; Caspar, Martial et Féray sous presse).

\section{Les outils liés à la transformation des matières textiles}

La production textile s'entend au sens large en contexte archéologique néolithique et recouvre aussi bien la corde, la cordelette ou le fil, les textiles cordés ou tissés ainsi que les filets, suivant les procédés employés pour la fabrication de ces différents biens.

Les fusaïoles

Les fusaïoles ne sont pas connues avant le Néolithique final dans cette région, alors que les premiers exemplaires apparaissent dès le Rubané en Allemagne et au Néolithique moyen, à partir du Cortaillod, sur les sites lacustres du Plateau suisse.

La fusaïole est un volant d'inertie destiné à alourdir le fuseau et à en faciliter la rotation au cours du filage. C'est l'objet le plus répandu de la culture matérielle associée à l'artisanat textile. Le fuseau permet de filer rapidement d'importantes quantités de fibres contrairement au filage effectué à main nue plutôt réservé à la fabrication de cordes et cordelettes en liber, plus épaisses et plus résistantes.

La variété typo-morphologique des fusaïoles contribue à préciser la qualité de la matière textile produite, car leur poids et leurs dimensions influent sur le mouvement de rotation qu'elles entraînent. Le moment d'inertie, la vitesse de rotation, la masse de la fusaïole ont des conséquences directes sur le diamètre des fils réalisés (Médard 2006 : 138-139). Les recherches menées sur les fusaïoles néolithiques indiquent qu'elles étaient conçues pour fabriquer des fils fins inférieurs à $1 \mathrm{~mm}$ de diamètre, selon une méthode qui pourrait être celle du filage au fuseau suspendu; ce procédé est particulièrement adapté aux fibres de lin ou de liber car la tension exercée sur elles en facilite le filage, par opposition au fuseau posé qui convient mieux au filage de la laine (Médard 2006 : 142143). Les fusaïoles mises au jour à Annoeullin, à Carvin et à Houplin-Ancoisne, toutes en terre cuite, ont des formes discoïde, conique, biconique ou encore hémisphérique (fig. 8).

Les pesons de métier à tisser Les fils néolithiques réalisés $\mathrm{au}$ fuseau étaient en majeure partie destinés au tissage. La présence de pesons implique la fabrication de tissu sur des métiers à tisser verticaux à poids. Sur ce type de métier, les fils de chaîne sont maintenus ensemble et tendus à l'aide des pesons. Ceux-ci sont adaptés à la qualité des fils sur lesquels ils exercent la traction et à des exigences fonctionnelles précises telles que le nombre de fils qui y sont attachés, leur diamètre et leur résistance (Médard 2006).

Différents types morphologiques de pesons, tous en terre cuite et sans décor, ont été mis au jour sur les occupations du Néolithique final du nord de la France. Les poids de métier à tisser trouvés à Carvin sont tous de forme tronconique à perforation horizontale (fig.8). Sur le site de RaillencourtSainte-Olle "le Grand Camp», qui domine la vallée de l'Escaut (fig. 1), le comblement d'une grande fosse circulaire comprenait un rejet de 74 pesons en terre cuite probablement issus du démantèlement d'un métier à tisser. Au total, les six fosses de ce site ont livré 128 pesons dont 72 entiers, tous de morphologie extrêmement standardisée, cylindrique à perforation verticale (Bostyn et Praud 2000).

Les pointes en os Parmi l'outillage en matière dure animale exhumé du site du "Marais de Santes" à Houplin-Ancoisne (étudié par Y. Maigrot ; Praud dir. 2007), figurent deux pointes façonnées sur fibula, l'une de suidé, l'autre appartenant à un grand carnivore. La première, complète et de $65 \mathrm{~mm}$ de long (fig.9), porte des traces d'usure peu étendue sur la pointe. Sur l'autre, brisée dans sa partie proximale, l'usure est très prononcée. L'observation au microscope atteste une utilisation liée au travail des fibres végétales, probablement comme aiguilles à filocher.

L'approche pluridisciplinaire appliquée à l'étude des sites de la vallée de la Deûle met en évidence l'existence incontestable d'un artisanat textile pratiqué au Néolithique final, dès le début du $\mathrm{III}^{\mathrm{e}}$ millénaire avant notre ère, à l'extrémité nord de la France. Dans le détail, les indices en sont relativement diversifiés bien que certains, plus ténus ou plus facilement sujets à caution, renvoient au manque relatif de données liée aux conditions de conservation dictées par le milieu naturel. En

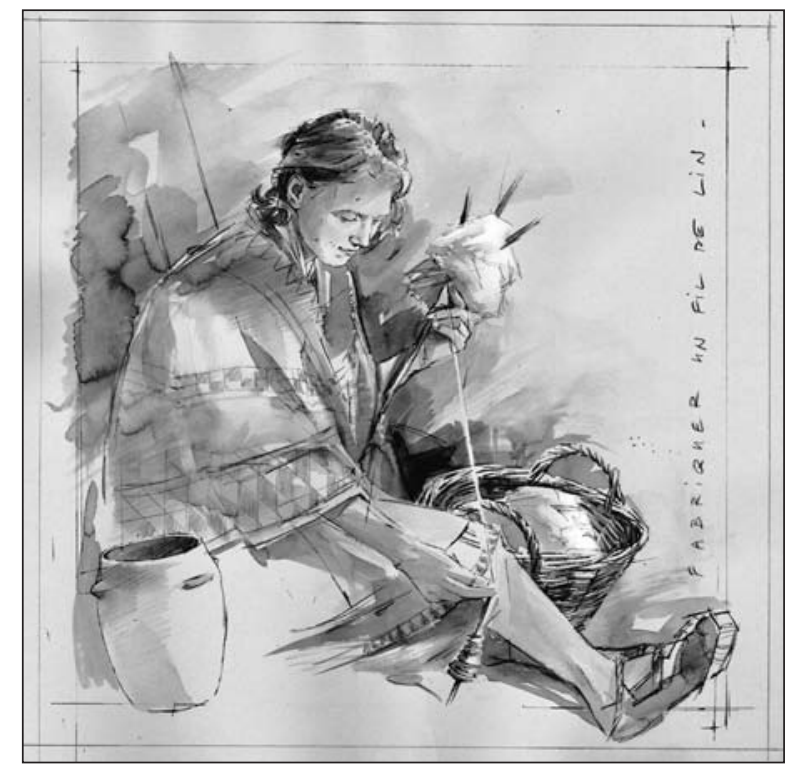

Fig. 7 - Le filage à l'aide d'un fuseau lesté d'une fusaïole (illustration B. Clarys). outre, certaines étapes de l'acquisition et de la transformation des matières textiles d'origine végétale sont difficiles à mettre en évidence, en particulier lorsqu'elles ne nécessitent pas d'outils ou alors un outillage en matériaux périssables.

D’après les données archéobotaniques, les ressources en liber et en fibres de plantes sauvages sont potentiellement disponibles dans l'environnement immédiat de tous les sites, que ce soit dans la forêt (chênaie mixte) qui couvre le versant, dans la ripisylve (aulne, saule) qui borde la Deûle ou encore dans le marais qui occupe le fond de la vallée (roseau, jonc). Les différentes 


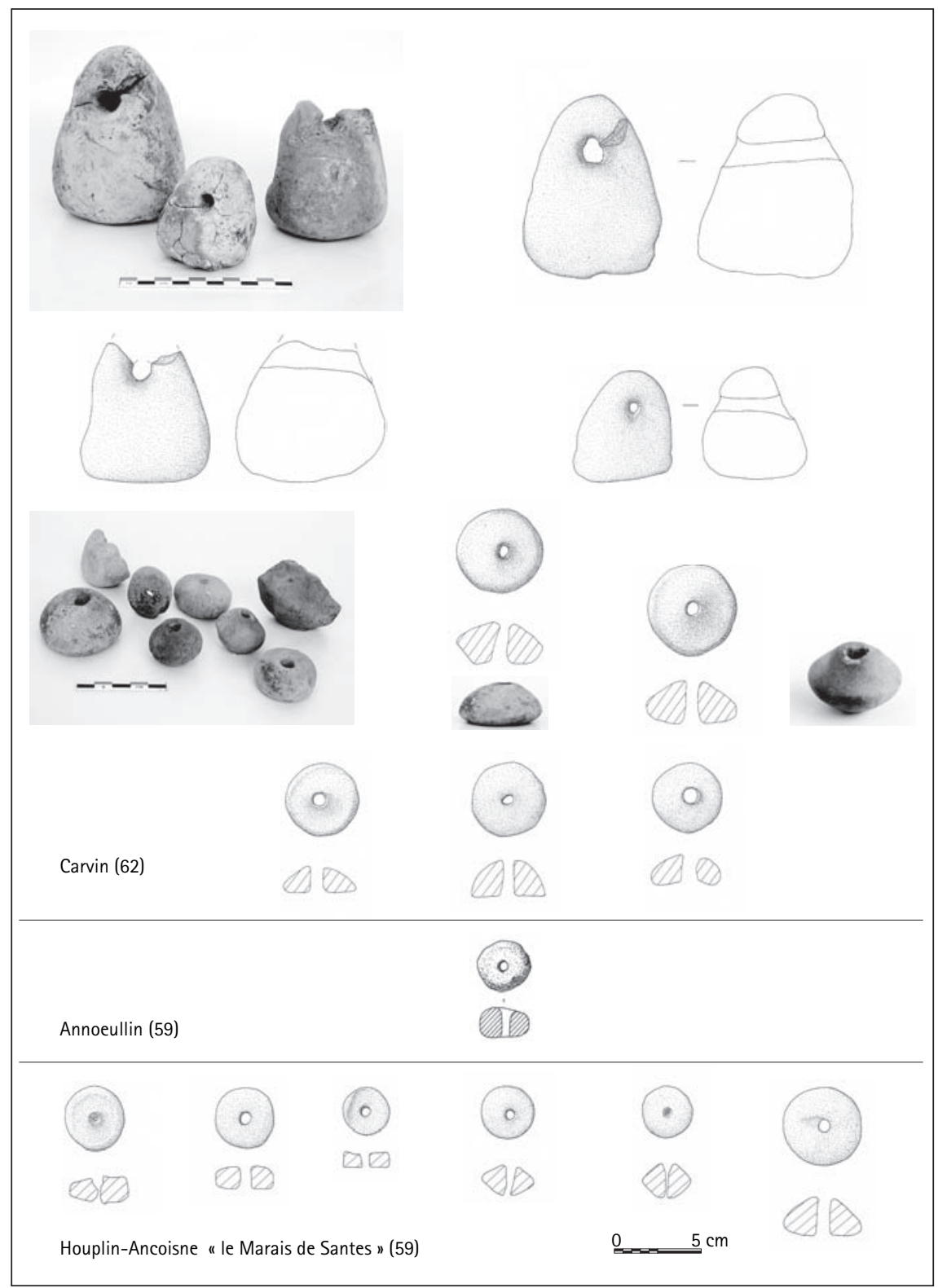

analyses montrent que nombre de ces espèces a d'ailleurs été exploité par les occupants de ces sites, en particulier à des fins architecturales ou alimentaires. Pour les plantes cultivées, le lin est attesté à Houplin-Ancoisne, ses graines étant particulièrement bien représentées "rue Marx Dormoy». S'il est bien un matériau emblématique de l'artisanat textile, il est aussi cultivé pour les qualités nutritives oléagineuses de ses graines. Le caractère contraignant et délicat de sa culture explique probablement que son utilisation textile réduite soit réservée visiblement à la production de fils et de tissus fins (Médard 2000).

L'hypothèse d'une aire de rouissage avancée pour la grande fosse étudiée sur le site de la "rue Marx Dormoy" serait l'unique indice de cette phase du traitement des fibres textiles. Rappelons que le rouissage peut se faire également en eau courante ce qui ne laisse évidemment aucune trace archéologique. En raison de ses effets polluants, la macération
Fig. 8 - Fusaïoles et pesons en terre cuite du Néolithique final des sites de Carvin, Annoeullin et Houplin-Ancoisne "Marais de Santes".

en fosse limiterait les émanations nocives comparée au rouissage pratiqué directement dans la rivière.

La transformation de la matière première textile est illustrée par trois types d'objets illustrant autant d'étapes différentes d'une, voire, deux chaînes opératoires : le filage, le tissage et le filochage.

La production de fil fin est attestée sur les quatre sites à travers l'omniprésence des fusaïoles en terre cuite destinées à lester les fuseaux. Leur grand nombre est sans doute révélateur de l'importance de l'activité de filage dans la vie quotidienne, comme le suggèrent les témoignages ethnographiques. Le fil fin peut servir à la fabrication de tissu mais aussi de filets, en particulier ceux utilisés pour la pêche (Médard 2006).

Le tissage est illustré par l'existence de pesons en terre cuite à Carvin et à Houplin-Ancoisne "rue Marx Dormoy". Leur distribution suggère que la fabrication de tissu sur métier à tisser vertical n'est pas générale. Cette activité est probablement exercée par un effectif plus réduit d'artisans. Sur la station lacustre néolithique de Delley-Portalban II (Suisse), les fusaïoles sont dispersées sur toute la surface du site, à l'intérieur et à l'extérieur des habitations, alors que la répartition des pesons reflète des emplacement réservés au tissage à l'intérieur des maisons (Médard 2000).

Deux pointes en os interprétées comme des aiguilles à filocher, renvoient à un autre aspect de la production textile. Elles ont été mises au jour au "Marais de Santes» à HouplinAncoisne et peuvent être mises en relation avec d'autres instruments découverts sur ce site et liés à la pratique de la pêche, en particulier un hameçon en ivoire et un poids de filet de pêche en craie. La fabrication de filets de pêche, à la différence des filets de portage ou de chasse plus résistants, peut exiger l'emploi de fil fin pour qu'ils soient moins visibles dans l'eau.

La répartition spatiale des traces d'artisanat textile semble indiquer, dans la limite que nous impose l'état de la documentation archéologique, une(des) chaîne(s) opératoire(s) géographiquement scindée(s), probablement au sein d'un territoire dont elle pourrait refléter l'organisation. La culture 


\section{E. Martial | Exploitation des végétaux et artisanat textile au Néolithique final}

du lin, à une extrémité de la chaîne, et le tissage à l'autre extrémité ne paraissent pas ubiquistes dans cette partie de la vallée de la Deûle, contrairement à la production de fil fin. La poursuite des recherches permettra de cerner plus précisément les modalités de cet artisanat dans le nord de la France où la production textile, en particulier linière, est particulièrement réputée de nos jours encore.

\section{Bibliographie}

Bostyn, F. et I. Praud. 2000. "Le site néolithique de Raillencourt-SainteOlle "le Grand Camp" (Nord)", in: Internéo 3, Actes de la Journée d'information du 2 décembre 2000. Paris : 119-129.

CASPAR, J.-P., P. FérAY et E. MARTIAL. 2005. «Identification et reconstitution des traces de teillage des fibres végétales au Néolithique», Bulletin de la Société préhistorique française, 102/4 : 867-880.

CASPAR, J.-P., E. Martial et P. Féray. Sous presse. "Le teillage des fibres végétales: pour une réinterprétation fonctionnelle d'outils en silex néolithiques", in: Actes du $26^{\text {e }}$ Colloque interrégional sur le Néolithique, INTERNÉO, 8-9 novembre 2003, Musée national d'histoire et d'art, Luxembourg (grand-duché de Luxembourg), Archeologia Mosellana.

Constantin, J. et F. FaldeAu. 1922. Histoire naturelle. Les plantes. Paris, Librairie Larousse.

FECHNER, K. 2000. "Reconstituer le paléoenvironnement à l'échelle humaine. Quelques résultats de l'archéopédologie sur le tracé occidental du train à grande vitesse en Wallonie (provinces de Hainaut et de Brabant). Actes de la Journée d'archéologie en province du Hainaut, Mons, 23 octobre 1999», Hainaut, $3:$ 87-101.

MARTIAL, E. et F. MÉDARD. 2007. "Acquisition et traitement des matières textiles d'origine végétale en préhistoire: l'exemple du lin", in: V. Beugnier et P. Crombe (éds), Plant Processing from a Prehistoric and Ethnographic Perspective / Préhistoire et ethnographie du travail des plantes. Oxford, John \& Erica Hedges Ltd (British Archaeological Report, International Series; S1718) : 67-82.

Martial, E., I. Praud et F. Bostyn. 2004. "Recherches récentes sur le Néolithique final dans le Nord de la France», in : M. VAN DER LINDEN et L. SALANOVA (dir.), Le troisième millénaire dans le nord de la France et en Belgique, Actes de la journée d'études SRBAP-SPF, 8 mars 2003, Lille. Paris, Société préhistorique française (Mémoire de la Société préhistorique française ; XXXV, Anthropologica et Praehistorica, 115) : 49-71.

Martial, E. et I. Praud, avec la collaboration de M. Boulen, S. Braguler, J.-P. Caspar, B. Clavel, S. Coubray, L. Deschodt, M.-F. Dietsch-Sellami, K. Fechner, C. Lehnebach et Y. Maigrot. Sous presse. "Un site palissadé du Néolithique final à Houplin-Ancoisne (Nord, France)", in: Actes du $26^{e}$ Colloque interrégional sur le Néolithique, INTERNÉO, 8-9 novembre 2003, Musée national d'histoire et d'art, Luxembourg (grand-duché de Luxembourg), Archeologia Mosellana.

MÉdARD, F. 2000. L'artisanat textile au Néolithique. L'exemple de DelleyPortalban II (Suisse): 2782-2462 av. J.-C. Montagnac, Ėditions Monique Mergoil (Préhistoire; 4).
MÉDARD, F. 2006. Les activités de filage au Néolithique sur le Plateau suisse. Analyse technique, économique et sociale. Paris, CNRS Éditions (CRA monographies; 28).

Praud, I. et E. Martial, avec la collaboration de V. Beugnier, L. Deschodt, R. LANGHOR, J. Lantolne et G. LouWAgie. 2000. "Une nouvelle occupation du Néolithique final dans la vallée de la Deûle, à Annoeullin (Nord)", in: Internéo 3, Actes de la Journée d'information du 2 décembre 2000. Paris : 131-142.

Praud, l. (dir.). 2007. Houplin-Ancoisne (59), "le Marais de Santes». Rapport de fouille archéologique, INRAP et SRA Nord - Pas-de-Calais, 2 volumes.

Praud, I., V. Bernard, E. Martial et R. Palau. 2007. "Un grand bâtiment du Néolithique final à Houplin-Ancoisne "le Marais de Santes" (Nord, France)", in: Actes du $27^{e}$ Colloque interrégional sur le Néolithique, 1 et 2 octobre 2005, Neuchâtel, Suisse, Cahiers d'archéologie romande, $108: 445-460$.

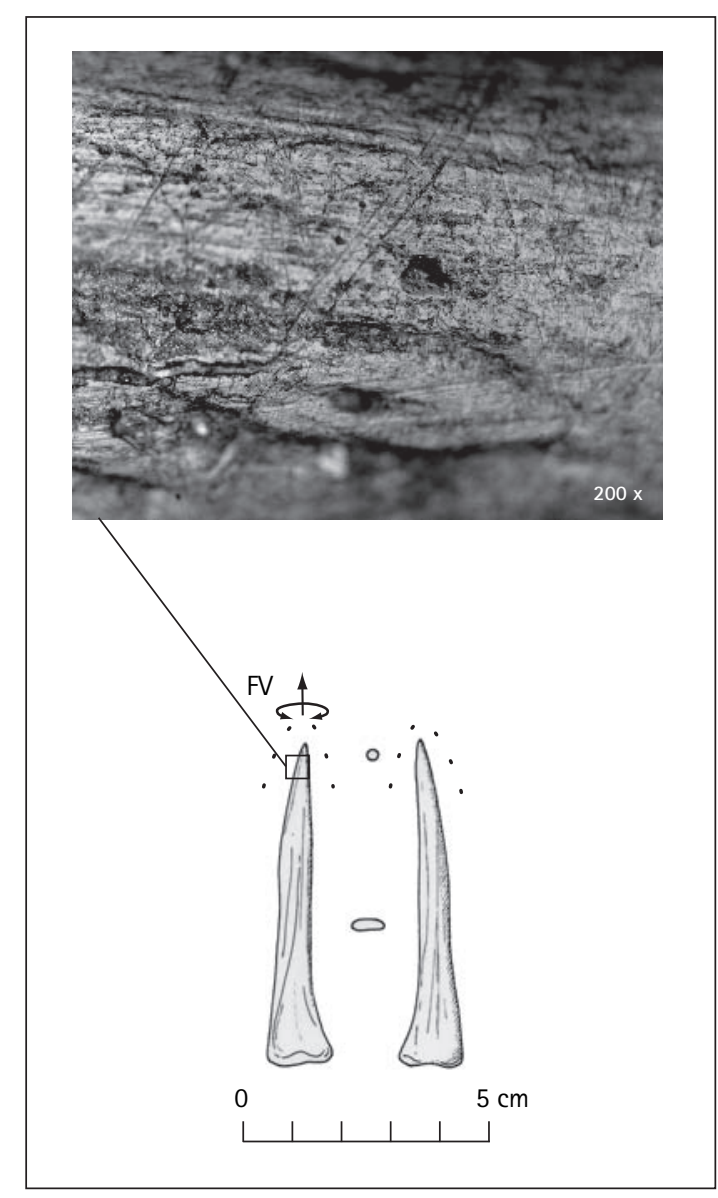

Fig. 9 - Pointe en os du site de Houplin-Ancoisne "Marais de Santes", utilisée pour le travail des fibres végétales (d'après Y. Maigrot). 